\title{
El downsizing como estrategia para la reorganización empresarial: ¿es factible en la era postpandemia COVID-19?
}

Downsizing as a strategy for business reorganization: is it feasible in the post-COVID-19 pandemic era?

1 Albert Joao Nieto Pacheco

Universidad de Guayaquil, Guayaquil, Ecuador

https://orcid.org/0000-0001-5296-5574

albert.nietop@ug.edu.ec

2 Ronny Pedro Carriel Sevillano

https://orcid.org/0000-0003-2484-1325

Universidad de Guayaquil, Guayaquil, Ecuador Ronny

carriels@ug.edu.ec

Artículo de Investigación Científica y Tecnológica

Enviado: 11/12/2021

Revisado: 26/12/2021

Aceptado: 28/01/2022

Publicado:05/02/2022

DOI: https://doi.org/10.33262/ap.v4i1.1.165

Nieto Pacheco, A. J., \& Carriel Sevillano, R. P. (2022). El downsizing como estrategia para la reorganización empresarial: ¿es factible en la era postpandemia COVID-19?. AlfaPublicaciones, 4(1.1), 372-381. https://doi.org/10.33262/ap.v4i1.1.165

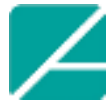

Ciencia Digital

ALFA PUBLICACIONES, es una Revista Multidisciplinar, Trimestral, que se publicará en soporte electrónico tiene como misión contribuir a la formación de profesionales competentes con visión humanística y crítica que sean capaces de exponer sus resultados investigativos y científicos en la misma medida que se promueva mediante su intervención cambios positivos en la sociedad. https://alfapublicaciones.com

La revista es editada por la Editorial Ciencia Digital (Editorial de prestigio registrada en la Cámara Ecuatoriana de Libro con No de Afiliación 663) www.celibro.org.ec

Esta revista está protegida bajo una licencia Creative Commons Attribution Non Commercial No Derivatives

4.0 International. Copia de la licencia: http://creativecommons.org/licenses/by-nc-nd/4.0/ 


\section{Palabras}

claves:.

downsizing, pandemia, estrategia, reorganización.

\section{Keywords:}

downsizing, pandemic, strategy, reorganization.

\section{Resumen}

El objetivo general de esta investigación se basa en analizar el downsizing como una estrategia para la reorganización empresarial y verificar su factibilidad en la post-pandemia. La metodología aplicada es de un diseño bibliográfico de tipo documental. El downsizing a pesar de no ser muy apreciada sobre todo en la actualidad por estar frente a las consecuencias de la pandemia por COVID-19 permite poder enfrentar estas situaciones externas de cambio debido a las restricciones económicas políticas y sociales impuestas por los países, así como también el poder permanecer activos en el mercado a pesar de las dificultades producto de la disminución de las actividades productivas; De igual manera, los directivos al conocer mejor y entender las ventajas que esta posee se puede lograr que las personas involucradas mejoren sus puntos de vista y acepten que los cambios organizacionales aplicados están hechos no solo para alcanzar las metas, sino también para lograr una satisfacción laboral entre los empleados, minimizando el estrés laboral, e incrementando los niveles de productividad. Asimismo, el downsizing ha permitido facilitar el manejo de las áreas de trabajo donde existían procesos complejos y que producto de la pandemia tuvieron que reducir sus operaciones, por lo tanto, al disminuir el uso de recursos para optimizar estos procesos se logra ejecutar actividades que permiten el manejo adecuado de las operaciones y aumente la rentabilidad que tanto se ha visto afectada por la presencia del Coronavirus.

\section{Abstract}

The general objective of this research is based on analyzing downsizing as a strategy for business reorganization and verifying its feasibility in the post-pandemic. The applied methodology is of a bibliographic design of a documentary type. Downsizing, despite not being highly appreciated, especially at present because it is facing the consequences of the COVID-19 pandemic, allows us to face these external situations of change due to the economic, political and social restrictions imposed by the countries, as well as also being able to remain active in the market despite the difficulties caused by the decrease in productive activities; In the same way, the managers, by knowing better and understanding the advantages that it has, it can be achieved that the people involved improve their points of view and accept that the applied organizational changes 
are made not only to achieve the goals, but also to achieve satisfaction. work among employees, minimizing work stress, and increasing productivity levels. Likewise, downsizing has made it possible to facilitate the management of work areas where complex processes existed and which, as a result of the pandemic, had to reduce their operations, therefore, by reducing the use of resources to optimize these processes, activities that allow the proper management of operations and increase profitability that has been so affected by the presence of the Coronavirus.

\section{Introducción}

La pandemia producto de la COVID-19 trajo consigo innumerables cambios a nivel mundial, desde las restricciones económicas, políticas, y sociales que generaron caos y procesos de adaptación en la sociedad. Estos cambios repercutieron de igual manera a nivel organizacional pues un gran porcentaje de las empresas tuvieron que detener sus actividades para poder cumplir con las exigencias impuestas por los países, y en muchos otros casos cesaron sus actividades por completo llegando al cierre de sus negocios.

Producto de esta crisis generada por el coronavirus las empresas que han logrado sobrevivir, se vieron en la obligación de evolucionar y cambiar sus estrategias de manera que pudieran subsistir durante la pandemia. Entre estos cambios se pueden mencionar los formatos de trabajo como el trabajo desde casa, el teletrabajo, y cambios en la estructura organizacional e inclusive la digitalización de muchos procesos (Hagemann \& Mayol, 2020).

Estas estrategias, asumidas por las empresas son parte de una reorganización que le han permitido no solo desde la pandemia sino en diversas etapas de la vida empresarial, lograr conservarse en tiempo y espacio y mantener al negocio funcionando. En este sentido de Marti (2021) indica que, es importante que las empresas cuenten con una buena planificación para realizar los cambios que consideren necesarios y así tener las ideas claras sobre el camino que desean seguir y las metas que necesitan alcanzar.

De la misma manera De Marti (2021) menciona que:

Resulta de vital importancia la reestructuración organizativa de las empresas con el fin de preparase a la nueva realidad socio-económica post-Covid. La pandemia ha conllevado la aceleración de la transformación digital y la necesidad de las compañías de adaptarse, en tiempo récord, a los nuevos modelos de negocio para lograr su supervivencia. 
Una estrategia que ha sido utilizada durante la pandemia es el downsizing, aplicada principalmente por las empresas con el propósito de ser más competitiva y en muchos casos para poder sobrevivir debido a falta de solvencia $u$ otros factores que provoquen la inestabilidad de la empresa e inclusive cuando se desea expandir el mercado o latitudes (Espinar, 2021).

En este sentido a través de esta investigación se pretende analizar el downsizing como una estrategia para la reorganización empresarial y verificar su factibilidad en la postpandemia. La metodología aplicada es de un diseño bibliográfico de tipo documental.

\section{Metodología}

La metodología aplicada en esta investigación se basa según menciona Palella \& Martins (2010) en un diseño bibliográfico basado en una revisión estructurada y profunda de material obtenido de diversas fuentes, para analizar los fenómenos y establecer relaciones con el tema investigado.

De igual manera se indica que el tipo de investigación es documental basada exclusivamente en la recopilación de información para obtener y analizar datos que provienen de las fuentes investigadas para profundizar y analizar temas de interés.

\section{Resultados y Discusión}

\section{Reorganización empresarial}

Esta ocurre por decisiones de la alta gerencia con el fin de lograr un nivel adecuado de competitividad por lo tanto realizan un nuevo diseño dentro de la estructura de la organización. De igual manera durante estos procesos de reorganización surgen cambios de puestos de trabajo y remociones de puestos, reducción de personal de manera que la dirección empresarial sea más fácil en momentos difíciles (Espinar, 2021).

De igual manera, menciona Cao (2017), que la reorganización dentro de la empresa son procesos que permiten el desarrollo organizacional por medio de intervenciones estratégicas para generar mayor eficacia y que el trabajo en cada área sea más efectivo.

La pandemia a nivel mundial generó que muchas empresas tomaran decisiones en muchos casos drásticas en cuanto a cambios sustanciales dentro de la organización producto de los constantes obstáculos que requerían de adaptación constante y también flexibilidad organizacional para cumplir los objetivos y alcanzar las metas planteadas.

Valencia (2018) indica que las organizaciones son sistemas abiertos, por lo tanto, es imposible que no se vean amenazadas y afectadas por medios externos, como las exigencias de mercado que cada día son más complejas, y actualmente se le ha sumado el impacto de la pandemia que generan barreras para continuar con la normalidad de las 
actividades, que ha forzado a las empresas buscar maneras de poder entender la dinámica presente en estos cambios.

\section{El Downsizing: estrategia empresarial}

El origen de este término surge durante los años 70 en Estados Unidos específicamente en el sector automovilístico debido a la necesidad que tenían para mantenerse en el mercado Alzamora \& Castro (2021), ya para los años 80's fue más sonado este término pues con este se utilizaba también el outplacement, el rightsizign entre otros.

Melgar (2011) menciona que este término se origina de la palabra "Down" que traducido al español significa disminuir o reducir, y era utilizada para mejorar la rentabilidad de las empresas (Quintana et al., 2012). Asimismo, se menciona que el downsizing también forma parte del estudio del comportamiento organizacional, así como también realiza análisis e los proceso contables y económicos y con especial énfasis en los recursos humanos.

Este término inicialmente se relacionó solo con reducción de personal, pero con el paso del tiempo y la importancia que se le ha dado a esta estrategia se han realizado ajustes, donde realizar un recorte de personal no es solo eso sino, una transformación necesaria para crear una visión diferente de la estructura organizacional, sus valores y cultura (Soria \& Herrero, 2016).

En este sentido Universidad de San Martín de Porres (2018) es su manual de estudios sobre Introducción a la Administración, menciona que más allá de los despidos masivo de empleados, existen tres tipos de estrategias downsizing y estas se reflejan en la tabla 1 .

\section{Tabla 1}

Tipos de estrategias basadas en Downsizing

\begin{tabular}{|c|c|}
\hline Tipo & Concepto \\
\hline $\begin{array}{l}\text { Reestructuración } \\
\text { numérica }\end{array}$ & $\begin{array}{l}\text { Es una estrategia a corto plazo la cual se basa en la reducción de numero } \\
\text { de efectivos. }\end{array}$ \\
\hline $\begin{array}{l}\text { Reestructuración } \\
\text { funcional }\end{array}$ & $\begin{array}{l}\text { Es una estrategia a mediano plazo con la cual se pretende lograr una } \\
\text { reestructuración de procesos; esto se realiza a través de diversos métodos } \\
\text { como: redefinición de tareas, eliminación de niveles, fusión de unidades y } \\
\text { supresión y reconversión de algunas funciones. }\end{array}$ \\
\hline $\begin{array}{l}\text { Reestructuración } \\
\text { estratégica }\end{array}$ & $\begin{array}{l}\text { Estrategia a largo plazo que tiene como objetivo la reconfiguración de la } \\
\text { organización y sus parámetros, con la cual se consideran aspectos como: } \\
\text { cambio de las responsabilidades, recomposición de la fuerza de trabajo, } \\
\text { implicación global, mejora continua y una revisión de la validez del } \\
\text { sistema de valores y normas de la organización. }\end{array}$ \\
\hline
\end{tabular}

Fuente: Universidad de San Martín de Porres (2018) 
Este tipo de reorganización empresarial permite mejorar y rediseñar la empresa en todos sus aspectos, en este proceso se readecúa la cantidad de empleados para que la empresa sea mas competitiva (Cuevas \& Beltrán, 2017).

Cuando se realiza una reducción de personal se aplican diversos recursos como despidos, jubilaciones anticipadas, para el caso de la reestructuración funcional se hacen cambios en los procesos de trabajo, en las actividades y se disminuyen las funciones, los niveles jerárquicos entre otros, en la reestructuración estratégica que es la que se realiza a largo plazo es la que busca cambiar los punto de vista de los empleados en referencia al downsizing y que puedan entender que esta es una estrategia para el mejoramiento continuo de la empresa (Laos, 2019).

Al habar del downsizing no se puede excluir tres conceptos que son importantes en las empresas modernas y que según Ciudad-Valls (2021) son los que se muestran en la tabla 2.

\section{Tabla 2}

\section{Conceptos claves en las empresas modernas}

\begin{tabular}{cl}
\hline Concepto & \multicolumn{1}{c}{ Descripción } \\
\hline & Este concepto menciona que las empresas poseen una mentalidad de ciclo de \\
fracaso, pues consideran que su mano de obra puede ser sacrificada y cambiada \\
Ciclo del fracaso \\
$\begin{array}{l}\text { y que a su vez existen empleados deficientes y poco motivados que no les } \\
\text { interesa la satisfacción del cliente. }\end{array}$
\end{tabular}

Este enfoque perseguido por las empresas es solo instrumental pues solo esta inmerso en otros aspectos, pero no en algo específico, es decir que los

Enfoque utilitarista directivos por ejemplo utilizan a sus empleados para lograr un objetivo en pro de la empresa y poco es considerado el desarrollo profesional del empleado.

Se acuñó para describir una situación en la que el empleado se ve totalmente limitado por el ritmo de la línea de producción y no tiene libertad, por ejemplo, para decidir cuándo desea tomarse un descanso o hacer una breve pausa en su

Dependencia trabajo. Estas formas tan restrictivas de organización del trabajo están muy

organizativa extendidas en las economías modernas, tanto en los sectores industriales como en los de servicios.

Fuente: Ciudad-Vallsm (2021)

\section{Características del Downsizing}

Esta estrategia posee tres características principales desde el punto de vista laboral que son las mencionadas por (Lievano, 2019).

- La intención de la estrategia

- El momento en el cual se pretende implementar la estrategia y, 
- Los efectos de esta sobre los procesos de trabajo y el personal de la empresa

De esta manera, es importante distinguir que el Downsizing debe ser diferenciado del declive ya que las razones pueden ser diferentes entre una empresa y otra. También esta estrategia permite visualizar los efectos que genera el downsizing en cuanto a la organización. (Lievano, 2019).

\section{Tipos de Downsizing}

Según Laos (2019) indican que esta estrategia se clasifica desde dos enfoques:

- "Downsizing reactivo: es cuando la reorganización dentro de la empresa se realiza debido a eventualidades que se presentan y son desfavorables, en este caso se requiere de actuar frente a un cambio es decir a dar respuesta a una amenaza, esta se realiza a corto plazo para poder enfrentar la situación que se les presente. Este surge en su mayoría frente a situaciones económicas desfavorables donde se deben realizar disminuciones en sus procesos productivos sin considerar los objetivos de la empresa".

- "Downsizing proactivo: esta reorganización se realiza de forma anticipada, es decir ya se tiene conocimiento de los cambios y se planifica creando criterios y acciones para lograr alcanzar la competitividad que se desea, generando cambios en el organigrama de la empresa en todos sus niveles “.

Es importante que los directivos posean criterios que permitan establecer estrategias de trabajo para establecer metodologías adecuadas para cumplir los objetivos establecidos.

\section{Ventajas del Downsizing}

Esta herramienta utilizada como estrategia dentro de las organizaciones es muy ventajosa, pues permite lograr con el mínimo de los recursos mejorar áreas de la empresa o inclusive toda la estructura organizacional. En este sentido Cortes (2021) menciona que el downsizing permite minimizar la burocracia que es la generadora de atrasos en los procesos, así como también facilitar las decisiones dentro de la empresa.

Así mismo el downsizing permite que en aquellas empresas rígidas, y estandarizadas puedan ser consideradas nuevas ideas para mejorar los servicios o darle un nuevo enfoque a una estrategia, lo que da paso al empowerment ya que al reducir algunas funciones jerárquicas los empleados gozan de poder y autoridad para participar en las mejoras dentro de la organización lo que les permite sentirse más responsables y ser más participativos, esto igualmente permite mejorar la comunicación producto de sistemas más flexibles y menos complejos donde fluye la comunicación por todos los departamentos de forma ordenada. 


\section{Desventajas del downsizing}

Para Alzamora \& Castro (2021), el downsizing presenta una serie de desventajas que afectan directamente a los empleados que son despedidos pues esto trae consigo el aumento de la tasa de desempleo, de la misma manera los trabajadores que quedan dentro de la organización sufren de estrés laboral por la presión de sentir que pueden ser parte del recorte de personal, lo que genera una pérdida del sentido de pertenencia, asimismo genera desconfianza dentro del personal.

De la misma manera se menciona que al eliminar algunos niveles jerárquicos los empleados pierden el horizonte pues se sienten sin un supervisor que les indique las tareas que deben cumplir, así como el resentimiento y problemas entre los trabajadores.

\section{El Downsizing y la satisfacción laboral}

Otro aspecto muy importante al aplicar la estrategia de downsizing es la satisfacción laboral pues esta es determinante en la productividad de la empresa, ya que si el downsizing produce un impacto negativo evidentemente la productividad disminuye, y en caso contrario la misma aumenta. La satisfacción laboral en palabras de Quintana et al. (2012) es:

"La actitud general de un empleado hacia su trabajo e incluye los sentimientos positivos o respuestas laborales que se refleja en los efectos que ocurren a través del tiempo en los trabajadores, en donde pueden experimentar diferentes grados de placer o descontento en el trabajo o con respecto a diversos aspectos del mismo". (p. 50)

En tiempos de pandemia por la Covid-19, han surgido innumerables reajustes laborales, donde en ocasiones se han cerrado empresas, y en otras se ha realizado reducción de personal generando grandes efectos psicosociales, enfermedades y estrés por parte de los despedidos y los que quedan dentro de las empresas cumpliendo sus funciones.

\section{Conclusiones}

- La pandemia provocada por el virus de la COVID-19, causó muchos impactos negativos en todos los niveles en el mundo. Para el caso de las organizaciones es importante que siempre se mantenga al equipo de trabajo motivado, y positivo y es que como todo ser humano es más fácil ser pesimistas en tiempos difíciles.

- Sin embargo, es fundamental que la fuerza laboral y los directivos estén siempre en comunicación para lograr entender los enfoques y las estrategias que se desean establecer para alcanzar las metas deseadas a nivel organizacional, así como también mantener la marca en el mercado, adaptándose y amoldando las exigencias de los clientes con el fin de poder satisfacerlos a todos. 
- El downsizing es una estrategia que ha sido criticada duramente desde que se adoptó para casos donde la reducción de personal era el principal cambio en la reorganización empresarial, pero al conocer mejor y entender las ventajas que esta posee se puede lograr que las personas involucradas mejoren sus puntos de vista y acepten que los cambios organizaciones aplicados están hechos no solo para alcanzar las metas organizacionales sino también para lograr una satisfacción laboral entre los empleados, minimizando el estrés laboral, e incrementando los niveles de productividad.

- De igual manera el downsizing ha permitido facilitar el manejo de las áreas de trabajo donde existían procesos complejos y que producto de la pandemia tuvieron que reducir sus operaciones, por lo tanto, al disminuir el uso de recursos para optimizar estos procesos se logra ejecutar actividades que permiten el manejo adecuado de las operaciones y aumente la rentabilidad que tanto se ha visto afectada por la presencia del Coronavirus.

- Además, se puede mencionar que es mas conveniente aplicar el donwsizing proactivo de manera que al establecer bien la planificación de lo que se desea lograr dentro de la organización y al aplicar esta estrategia será mucho más fácil alcanzar los objetivos deseados pues se tiene más claro que camino se debe seguir para lograr mayor productividad en la empresa.

\section{Referencias Bibliográficas}

Alzamora, J., \& Castro, K. (2021). El Downsizing en la cadena de Gimnasios "Mundo Fitness" de la ciudad de Trujillo 2019. Trujillo, Perú: Trabajo especial de grado de la Universidad Privada Antenor Orrego para optar el título de Licenciado en Administración.

Cao, A. (2017). Los efectos de la Reforma Energñetica 2013 en la reestructuración Administrativa del Corporativo de PEMEX y Pemex Exploración y Producción. México: Foro Energético del Programa de Energia del Colegio de México.

Ciudad-Valls, I. (05 de febrero de 2021). El downsizing y la Covid-19 supervivientes y despedidos.

Cortes, N. (15 de abril de 2021). La estrategia del downsizing empresarial.

Cuevas, H., \& Beltrán, L. (2017). Reestructuración administrativa y financiera de la empresa REYCLO SAS. Bogotá, Colombia: Trabajo especial de grado de la Pontificia Universidad Javeriana.

De Marti, I. (14 de abril de 2021). La reestructuración de la empresa para adaptarse a la era post-Covid. 
Englobally Latinoamérica. (2019). Downsizing, estrategia de estructuración empresarial.

Espinar, J. (2021). Plan de rediseño organizacional de la empresa Cielo y Estilo S.A. Guayaquil, Ecuador: Trabajo especial de grado de la Universidad Católica de Santiago de Guayaquil para optar al título de Licenciada en Psicología Organizacional.

Hagemann, B., \& Mayol, F. (31 de agosto de 2020). La nueva organización post-COVID.

Laos, M. (2019). Gestión del Downsizing y el outplacement en la empresa cosmos agencia marítima sac, Callao 2019. Callao, Perú: Trabajo especial de grado de la Universidad Cesar Vallejo para optar al título de Licenciada en Administración.

Lievano, S. (2019). Reducción de personal-Downsizinf. ¿Parte del problema o de la solución? Cajica: Ensayo de la Universas Militar Nueva Granada.

Palella, S., \& Martins, F. (2010). Metodología de la investigación cuantitativa. Caracas, Venezuela: FEDUPEL, Fondo Editorial de la Universidad Pedagógica Experimental Libertador.

Quintana, A., Guillén, M., González, J., \& Cuevas, T. (2012). Estrategias del downsizing en las maquiladoras de Ciudad Juárez, Chihuahua, México. European Scientific Journal 8(27), México.

Soria, C., \& Herrero, A. (2016). Influencias de la estrategia de downsizing sobre la estructura de la empresa. Revista Brasileira de Gestäo de Negocios 19(63), 118132.

Universidad de San Martín de Porres. (2018). Introducción a la administración.

Valencia, L. (2018). Facilitadores y obstaculizadores de cambio asociados a un proceso de reestructuración organizacional en una empresa del sector industrial de la ciudad de Medellín. Trabajo especial de grado de la Universidad EAFIT para optar al título de Magister en desarrollo humano organizacional.

\section{Ciencia Ligital


El artículo que se publica es de exclusiva responsabilidad de los autores y no necesariamente reflejan el pensamiento de la Revista Alfa Publicaciones.

\section{Ciencia}

El artículo queda en propiedad de la revista y, por tanto, su publicación parcial y/o total en otro medio tiene que ser autorizado por el director de la Revista Alfa Publicaciones.
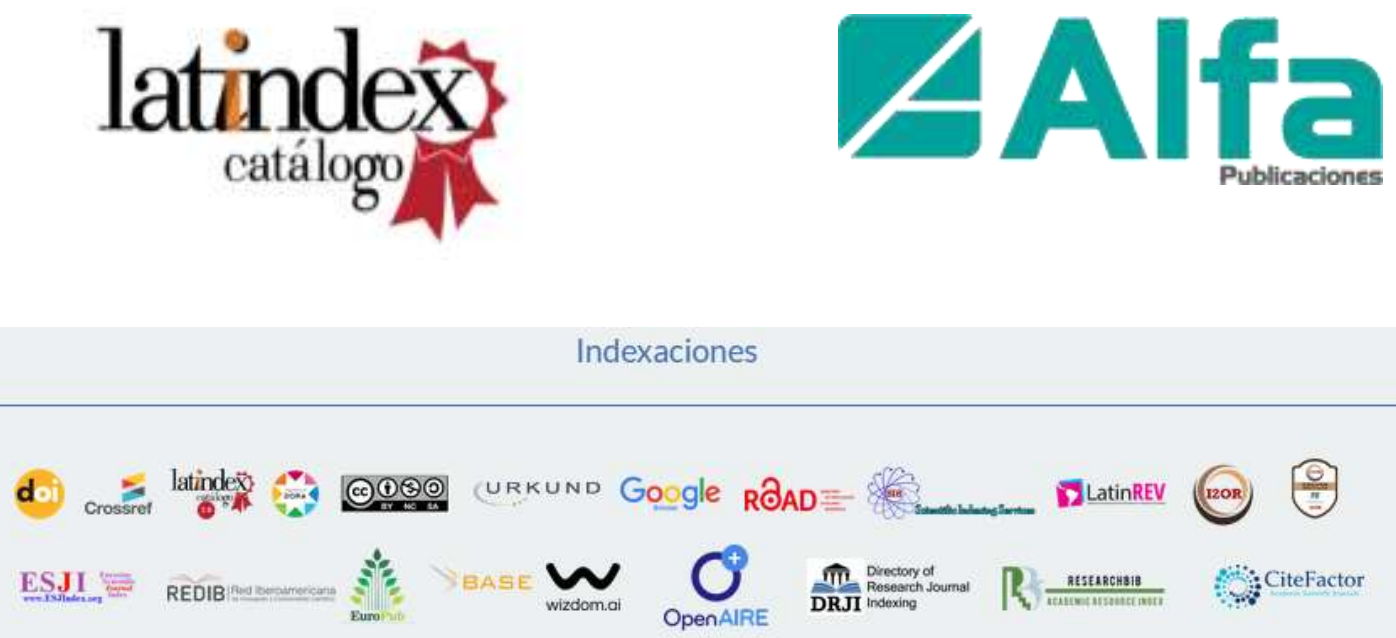